\title{
Simple Model for Cathode Coupling Voltage Versus Background Pressure in a Hall Thruster
}

\author{
Sarah E. Cusson, ${ }^{*}$ Benjamin A. Jorns, ${ }^{\dagger}$ and Alec D. Gallimore ${ }^{\ddagger}$ \\ University of Michigan, Ann Arbor, MI, 48109, USA
}

\begin{abstract}
A simple model is developed to capture the effect of background pressure on the cathode coupling voltage in a Hall effect thruster. The underlying hypothesis of this investigation is that the changes in cathode coupling voltage drop are dominated by the potential drop from the cathode exit to the thruster plume. This drop in turn is driven by the onset of anomalously high resistivity, resulting from ion acoustic turbulence. A one-dimensional model, based on quasi-linear formulation, is presented for how the growth of this ion acoustic turbulence drives the potential drop, and how this voltage drop depends on neutral density in the plume. Predictions from this model are compared to experimental data from the SPT-100 Hall thruster and it is shown that there is good agreement within uncertainty to the experimental data. This suggests that the facility effects on the cathode coupling voltage are largely driven by the ion acoustic turbulence in the plume of the hollow cathode. The results are then discussed in the context of performance effects on Hall thrusters.
\end{abstract}

${ }^{*}$ Ph.D. Candidate, Department of Aerospace Engineering, AIAA Student Member

${ }^{\dagger}$ Assistant Professor, Department of Aerospace Engineering, AIAA Senior Member

${ }^{\ddagger}$ Robert J. Vlasic Dean of Engineering, Department of Aerospace Engineering, AIAA Fellow 


\section{Nomenclature}

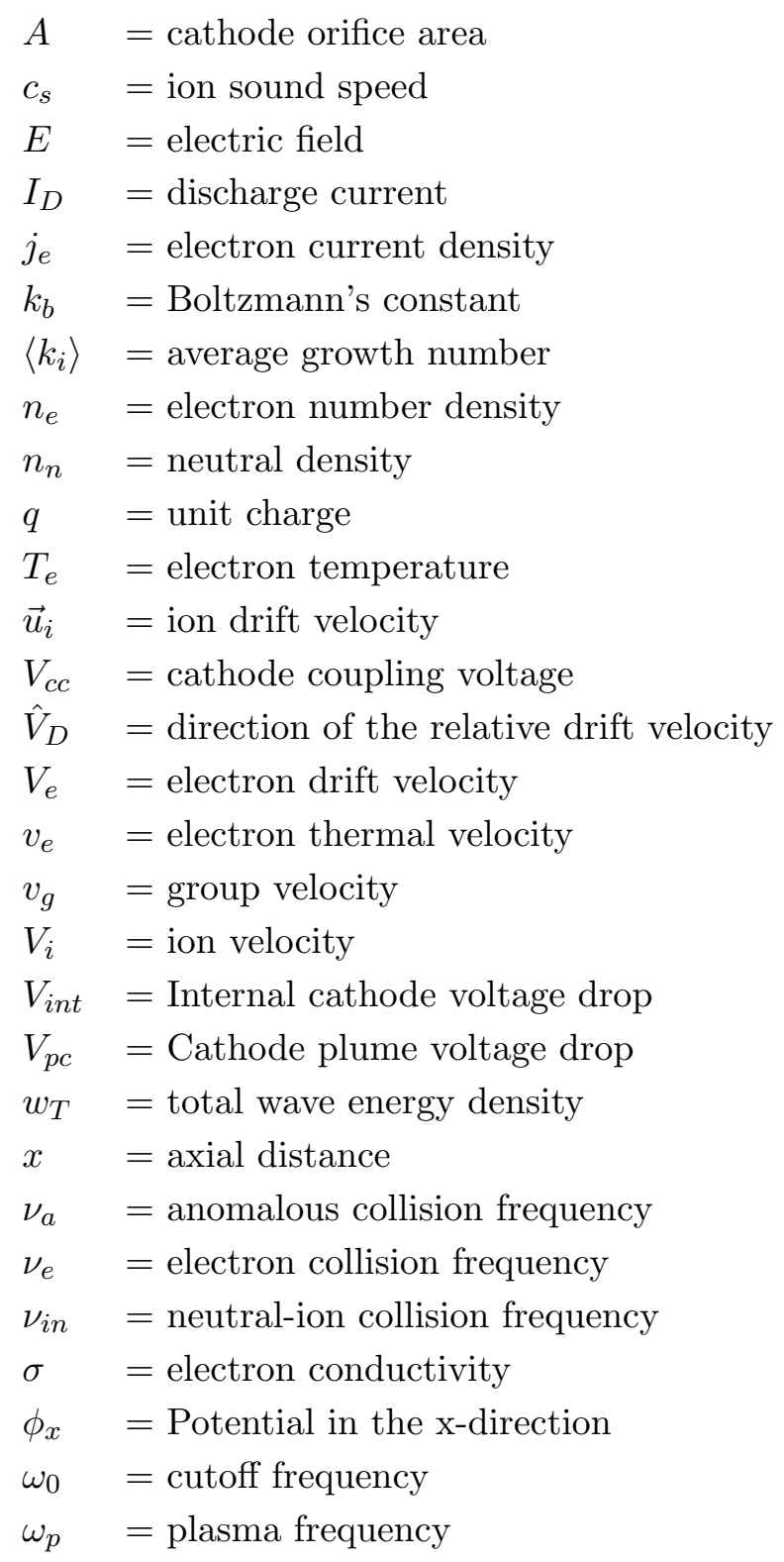

\section{Introduction}

Hall thrusters are a form of electric propulsion that use a crossed electric and magnetic field to ionize neutral gas and then accelerate it. They have decades of flight history in Earth orbit, and recent improvements in their lifetimes and efficiencies ${ }^{1,2}$ have increased their use in the commercial and government spacecraft sectors. ${ }^{3-5}$ However, a known issue with these devices is that their performance on-orbit is different than their performance during ground testing. This poses a significant risk for developing this technology, raising questions about the validity of the current standard practice of using ground test results for qualifying these systems for flight. The prevailing consensus is that the difference in performance largely can be attributed to so-called facility effects, i.e. differences in the test environment related to the presence of confining walls and limited pumping capacity as compared to the space environment. With this in mind, there was a concerted effort 
in the 1990s and early 2000s to develop standard practices for trying to replicate the space environment as closely as possible in ground test facilities. These best practices mostly centered on the problem of finite facility pressure. The goal was to establish an acceptable facility pressure for testing. These standards were informed by detailed pressure studies ${ }^{6,7}$ on the then most-flown Hall thruster, the SPT-100, a $1.5 \mathrm{~kW}$ device. It was thought that neutral gas ingestion due to finite facility pressures accounted for changes in performance. In order to ensure that neutral gas ingestion did not affect performance measurements, a facility pressure limit of $3 \times 10^{-5}$ torr was decided upon. ${ }^{7}$ This was the pressure at which the ingested gas was on the order of the uncertainty in the mass flow measurements.

Subsequent investigations have shown that the canonical presentation of pressure effects did not fully capture the impact. ${ }^{8-13}$ Moreover, these effects appears in all types of configurations including thrusters with externally mounted cathodes, with internally mounted cathodes, and magnetically shielded thrusters. Due to this extensibility, these effects are critical to understand. While the classical theory suggested that neutral ingestion was the dominant effect resulting in increased performance, there appear to be more nuanced but insidious effects that can occur even below the pressure level suggested by Randolph ${ }^{7}$ that can not be explained by a simple ingestion model. Parameters known to be affected are the location of the acceleration region, thrust and cathodecoupling voltage. In general, the acceleration region moves axially downstream, ${ }^{10}$ the thrust decreases ${ }^{8}$ and the cathode-coupling voltage increases in magnitude ${ }^{9}$ with decreasing background pressure. Accordingly, this affects the efficiency and specific impulse of each of the thrusters in ways not predicted by the simple ingestion model. Additionally, thruster stability is impacted by pressure effects. ${ }^{9}$

Furthermore, it is unclear whether trends observed during the pressure studies continue to hold when the pressure decreases beyond the limits of ground test facilities. A more recent study on the SPT-100, for example, revealed that even when testing was done below the published recommendation of $3 \times 10^{-5}$ torr, the thruster was still sensitive to changing background pressure. ${ }^{14}$ Generally, these studies attempt to empirically characterize the trends of impacted parameters with decreasing pressure and then extrapolate them to a vacuum environment. However, this technique is quite limited and it is not known if extrapolation is actually a valid technique. This has a large implication on translating ground data to on-orbit performance. In order to truly mitigate this effect, there is a need not only for empirical trends but a first-principles understanding. To this end, multiple theories behind the ground facility-thruster interactions have emerged. Frieman et $\mathrm{al}^{15,16}$ have looked at the role of the conducting wall in facility effects and Crofton suggested that the increase in thrust could be due to charge exchange. ${ }^{17}$ However, the fundamental manifestation of these effects still remains unidentified. In light of this, the need is apparent for continued development of a first-principles understanding of facility effects.

The goal of this paper is to study one of these effects: the change in cathode coupling voltage as a function of pressure. Changes in cathode coupling voltage are critical to thruster performance as the coupling voltage is a loss mechanism for the voltage utilization. Any voltage used to couple the cathode electrons to the thruster plume is not used to accelerate the plume ions, therefore decreasing the efficiency of the thruster. This effect is relatively small, only believed to be $1 \%$ of the total acceleration voltage, however a change in cathode coupling voltage may also result in a change of the boundary conditions for the acceleration region. Experimentally ${ }^{18}$ it has been shown that the magnitude of the cathode to ground voltage generally decreases with increasing facility pressure. While cathode to ground is not strictly the coupling voltage, it is generally considered a good indicator of changes in this voltage. This is because the other portion of the cathode coupling 
voltage, ground to plasma potential, is thought to vary to a smaller extent than cathode to ground

voltage. Spektor investigated facility effects on Hall thrusters through cathode coupling. ${ }^{19}$ The results showed that through electron-neutral collisions, the trends in thrust were recovered as a function of background pressure for both internally and externally mounted cathodes. However, in order to capture this result, the required magnetic fields were an order of magnitude too small. Thus, the need is still apparent to develop a first-principles understanding of this. The goal of this work is to explore a new hypothesis for what drives this cathode coupling and its subsequent dependence on facility pressure.

The central hypothesis of this work is that the cathode coupling voltage is driven by anomalous resistivity in the plume of the hollow cathode. This resistivity, in turn, depends on the neutral density. Until recently, Hall thruster and hollow cathode models have relied on an empirical anomalous collision frequency to model electron transport. ${ }^{20,21}$ More recent theoretical work has shown that by implementing a self-consistent model that includes the ion acoustic turbulence (IAT) in the cathode plume, the appropriate scaling for the anomalous resistivity can be recovered. ${ }^{22,23}$ The ion acoustic waves are driven by the electron kinetic energy through inverse Landau damping. Jorns et $\mathrm{al}^{22}$ showed that the electrons transfer their drift energy to the wave and that energy then gets redistributed to the internal energy (temperature) of the electron and ion fluids. This process has been confirmed experimentally: probe measurements verified the presence of ion acoustic turbulence in the plume of a hollow cathode and showed that ion temperature is correlated with wave energy, as predicted by the self-consistent model. ${ }^{22,24}$ The ion acoustic instability is damped by both ion Landau damping and ion-neutral collisions. Of particular interest here is the dependence on the ion-neutral collision frequency, which is proportional to the neutral density. As discussed above, increasing background pressure (increasing neutral density) results in a lower coupling voltage. Based on this instability, which has been shown to exist in hollow cathode plumes, the damping of the wave should have an inherent dependence on pressure. This implies that electrons are giving up less kinetic energy to the wave and, as a result, are more easily able to connect to the Hall thruster plume. The goal of this work is to show that increased background pressure damps the ion acoustic instability in the cathode plume and reduces the cathode coupling voltage.

To explore this effect, this paper is organized as follows. First, we employ a quasi-linear formulation to develop a one-dimensional model for the cathode coupling voltage in the plume as a function of neutral density. Second, using experimentally informed inputs into the model, agreement is found with experimental data from the SPT-100 pressure studies. Third, the results are discussed in the context of experimental uncertainty. Finally, concluding remarks about the implications of the results are made.

\section{Development of One Dimensional Model for Cathode Coupling Voltage}

This section develops the theory of how the cathode coupling voltage is influenced by the presence of ion acoustic turbulence in the cathode plume and how, in turn, changes in background pressure influence the growth of the wave.

As seen in Figure 1a, the Hall thruster can be split into two parts: the cathode and the anode. The cathode is an electron source for the thruster. Here, we focus specifically on hollow cathodes. Generally, they have a thermionic emitter that produces electrons necessary for ionization in the thruster channel. A voltage drop is necessary to force the electrons to travel from the cathode to 
the Hall thruster beam. This voltage drop is referred to as the coupling voltage and has been shown to vary with background pressure. ${ }^{7}$

Additionally, these thrusters have an inherent voltage drop that is set by the operator. The voltage drop necessary to pull electrons from the cathode to the anode makes up part of this total discharge voltage. The other portion is used to accelerate the ions. Since the ultimate velocity of the ions is proportional to the voltage used to accelerate them, any voltage used to couple the electrons to the anode is a loss mechanism. Specifically, the ultimate velocity of the ions is proportional to $\sqrt{V_{D}-V_{C C}}$, as seen in Figure 1b. Finally, since the total thrust of the device is proportional to the ions' velocity $\left(T \propto \sqrt{V_{D}-V_{C C}}\right)$ an increase in the voltage necessary for coupling electron will result in a decrease in thrust. Therefore, it is expected that a variation of the cathode coupling voltage with background pressure variation would also result in a variation in the total performance (thrust and efficiency) with background pressure. In order to capture this result, we develop a model to determine the dependence of cathode coupling voltage on background pressure.

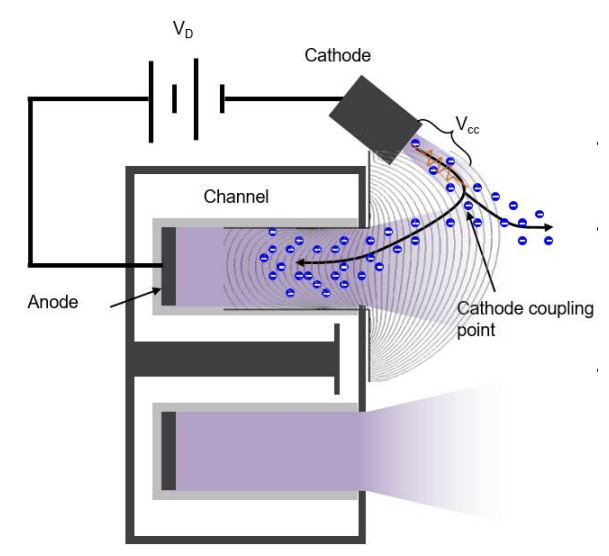

(a) A graphic showing the path of electrons in a Hall thruster. Electrons born in the cathode follow the magnetic field lines to the thruster plume.

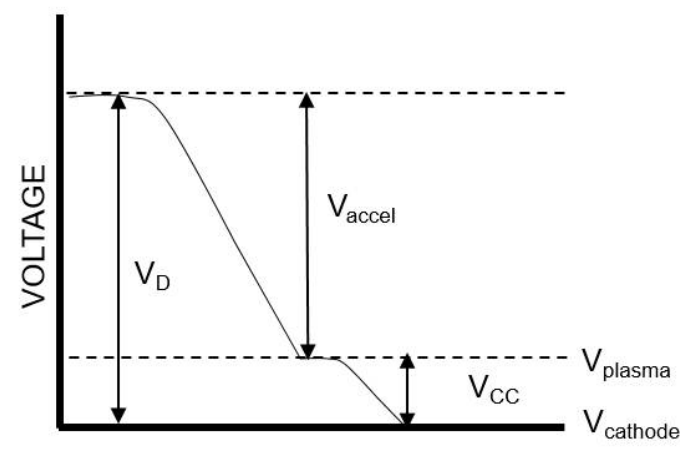

(b) A visual of the voltage drop in the plume. The cathode coupling voltage is an efficiency loss mechanism as it is not used to accelerate the ions.

Figure 1

To start, we break the cathode coupling voltage into two components,

$$
V_{c c}=V_{i n t}+V_{p c}
$$

where $V_{\text {int }}$ is the voltage drop from the cathode emitter to the cathode exit plane and $V_{p c}$ is the voltage drop from the cathode exit to the thruster plume as seen in Figure 2. Each one of these contributes to the total cathode coupling voltage, but we make a key assumption that $V_{\text {int }}$ is insensitive to facility neutral density. The reason for this is that interior to the cathode, the background neutral density in the facility is orders of magnitude smaller than the neutral density due to propellant flow through the cathode. For example, taking the SPT-100 cathode flow rate of $0.3815 \mathrm{mg} / \mathrm{s}$ and insert diameter of $6.3 \mathrm{~mm}$ and a modeled neutral temperature of $2000 \mathrm{~K}$, the pressure internal to the cathode is calculated to be 0.75 Torr. ${ }^{6,21,25}$ This is between 5 and 7 orders of magnitude higher than the facility background pressure. Therefore, it is assumed that $V_{\text {int }}$ is independent of background pressure. However, the same assumption is not true in the cathode plume. Modeling has shown that within one centimeter of the cathode exit the neutral density has dropped over 


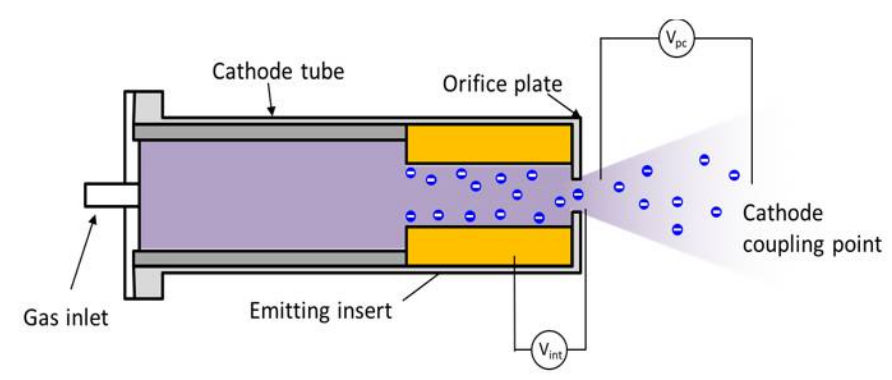

Figure 2: A schematic of the cathode in a Hall thruster. There are two components to the coupling voltage: the internal voltage drop inside the cathode and the voltage drop inside the plume.

4 orders of magnitudes. ${ }^{20}$ Since the density falls as the inverse of distance squared, the ratio of the density due to cathode flow and the density due to background neutrals is within one to two orders of magnitude of each other very close to the cathode exit plane. Seeing as these densities are comparable, it is expected that the cathode plume is susceptible to background pressure effects.

Based off these considerations, we concentrate solely on $V_{p c}$. In order to facilitate this discussion, we make some key assumptions. First, this model assumes that electrons flow from the cathode to the plume along magnetic field lines. Notably, previous modeling has shown that electrons have no appr1eciable mechanism of cross field transport in the hollow cathode plume, ${ }^{26}$ validating this assumption. This allows us to consider the electron dynamics as unmagnetized only moving along magnetic field lines. Therefore, this model is one dimensional along the magnetic field line on cathode centerline, as seen in Figure 1a. With this being the case, we can invoke a one-dimensional Ohm's law. We also make the assumption that the electron temperature is constant in space. Ohm's law can be thus written as,

$$
j_{e}=\sigma\left(E+\frac{k_{b} T_{e}}{q n_{e}} \nabla n_{e}\right)
$$

where $j_{e}$ is the electron current density, $\sigma$ is the electron conductivity, $E$ is the electric field, $q$ is the unit charge, $n_{e}$ is the electron number density, $k_{b}$ is Boltzmann's constant and $T_{e}$ is the electron temperature. This expression relates current density to the forces in the plume, specifically electric field and pressure gradient forces. The electron conductivity is known to be $\sigma=\frac{n_{e} q^{2}}{m_{e} \nu_{e}}$, where $\nu_{e}$ is the electron collision frequency. This key parameter can now be evaluated and related back to Eqn. 2.

In the hollow cathode plume, it has been shown that the electron resistivity cannot be described by classical electron transport equations. ${ }^{22,23}$ The electron resistivity is in fact orders of magnitude higher than what can be explained by classical effects, such as electron-ion collisions. In order to account for this effect, we include an anomalous term in the electron resistivity. With this in mind, the collision frequency can be broken out into multiple terms, i.e. $\nu_{e}=\nu_{e i}+\nu_{e n}+\nu_{a}$ where the first term is electron-ion collision, the second term is electron-neutral collisions, and the third term is the anomalous electron collision frequency. In order to properly capture the electron dynamics, this anomalous collision frequency is generally three orders of magnitude higher than the other two terms. ${ }^{26}$ Therefore, in this model, only the anomalous frequency is considered in the equation. Rearranging Eqn 2 for the potential drop and making the stated simplifications,

$$
-\nabla \phi_{x}=j_{e} \frac{m_{e} \nu_{a}}{n_{e} q^{2}}+\frac{k_{b} T_{e}}{q n_{e}} \nabla n_{e}
$$


In order to solve this equation, we need a model for the anomalous collision frequency. One prevailing theory in cathode plumes is that the anomalous collision frequency can be attributed to wave-particle interactions of the ion acoustic type. Sagdeev ${ }^{27}$ suggested the following scaling for the anomalous collision frequency,

$$
\nu_{a}=\alpha \omega_{p e} \frac{W_{T}}{T_{e} n_{e}}
$$

Here, $\omega_{p e}$ is the plasma frequency for electrons, $W_{T}$ is the total wave energy density, and $\alpha$ is a constant. The parameter $\alpha$ was empirically determined to be about $10^{-2}{ }^{27}$ In order to determine the total wave energy, the wave energy equation suggested by Jorns et $\mathrm{al}^{22}$ is used in one dimension. This model for the development of the wave energy has been shown to be self-consistent and properly capture the anomalous electron frequency in the cathode plume. It relies on including the ion acoustic turbulence which is thought to play a dominant role in this region of the plasma. The energy is seen to be a balance between convection, ion Landau damping, neutral damping, and inverse electron Landau damping. Thus,

$$
v_{g} \nabla \cdot W_{T}=W_{T} \omega_{0}\left[\frac{\pi}{2}\right]^{1 / 2}\left[\frac{V_{e}-V_{i}-c_{s}}{v_{E}}-\left(\frac{T_{e}}{T_{i}}\right)^{3 / 2} e^{-T_{e} / 2 T_{i}}-\frac{\nu_{i n}}{\omega_{0}}\right]
$$

where $v_{g}$ is the group velocity, $\omega_{0}$ is a cutoff frequency, $V_{e}$ is the electron drift velocity, $V_{i}$ is the ion velocity, $c_{s}$ is the ion sound speed, $v_{e}$ is the electron thermal speed, and $\nu_{i n}$ is the ion-neutral collisions frequency. Assuming that $V_{e} \gg V_{i}, c_{s}$ and rearranging, it can be seen that,

$$
\begin{array}{r}
\frac{\nabla W_{T}}{W_{T}}=\left\langle k_{i}\right\rangle \\
\left\langle k_{i}\right\rangle=\frac{\omega_{0}}{v_{g}}\left[\frac{\pi}{2}\right]^{1 / 2}\left[\frac{V_{e}}{v_{E}}-\left(\frac{T_{e}}{T_{i}}\right)^{3 / 2} e^{-T_{e} / 2 T_{i}}-\frac{\nu_{\text {in }}}{\omega_{o}}\right]
\end{array}
$$

where $\left\langle k_{i}\right\rangle$ is an average growth number. Eqn. 7 shows that the average growth number of this turbulence is dependent on the neutral density. This comes into the equation through $\nu_{i n}$. If the background neutral density is an appreciable percentage of the cathode neutral density, as is expected in the cathode plume, then this average growth number is sensitive to facility pressure.

Equation 6 is an ordinary differential equation for the propagation of the ion acoustic waves. We reduce this equation to one dimension along the streamline, subject to our assumptions. The physical constants here naturally vary as a function of position. However, it has been found empirically $^{28}$ that for a 100 -A cathode, the average wave growth is constant in space. Therefore, we can evaluate at one point and easily integrate Eqn. 6 to find:

$$
W_{T}=W_{T, 0} \exp \left(\left\langle k_{i}\right\rangle\left(x-x_{o}\right)\right)
$$

This can then be incorporated into Eqns 3 and 4 to find:

$$
-\nabla \phi_{x}+\frac{k_{b} T_{e}}{q n_{e}} \nabla n_{e}=j_{e} \frac{m_{e} \alpha \omega_{p e} \exp \left(\left\langle k_{i}\right\rangle\left(x-x_{o}\right)\right)}{T_{e} n_{e}^{2} q^{2}}
$$

Assuming that $j_{e}=n_{e} u_{e} q$ and directly integrating Eqn. 9, the cathode coupling voltage in the plume of the cathode is found to be,

$$
\Delta V_{p c}=\left(\frac{\alpha m_{e} V_{e} \omega_{p e} W_{T(0)}}{\left\langle k_{i}\right\rangle T_{e} n_{e} q}\right) \exp \left(\left\langle k_{i}\right\rangle\left(x-x_{o}\right)\right)+\frac{k_{b} T_{e}}{q} \log \frac{n_{e}}{n_{e, 0}}
$$


where $V_{p c}$ is the voltage drop in the plume as seen in Figure 2. To get the full coupling voltage, the voltage drop inside the cathode must also be included. That will not be considered here as $V_{\text {int }}$ is constant with varying background pressure and we are only concerned with relative changes. Additionally, since the drop-off of electron density with pressure is unchanged, the change in cathode-coupling with pressure is seen to be,

$$
\Delta V_{p c}=\left(\frac{\alpha m_{e} V_{e} \omega_{p e} W_{T(0)}}{\left\langle k_{i}\right\rangle T_{e} n_{e} q}\right) \exp \left(\left\langle k_{i}\right\rangle\left(x-x_{o}\right)\right)
$$

Finally, we assume a form for the energy density of $W_{T(0)}=\delta n_{0} T_{e}$. Here, $\delta \ll 1$ in order to introduce some initial thermal fluctuations required to excite the mode. This leaves

$$
\Delta V_{p c}=\left(\frac{\delta \alpha m_{e} V_{e} \omega_{p e}}{\left\langle k_{i}\right\rangle q}\right) \exp \left(\left\langle k_{i}\right\rangle\left(x-x_{o}\right)\right)
$$

Equation 14 shows the dependence of coupling voltage on background pressure. Qualitatively, it shows that an increase in pressure would result in damping of the waves which, in turn, would reduce the coupling voltage. An increase in pressure would result in a decrease in average wave growth number, $\left\langle k_{i}\right\rangle$, as the neutral damping term, $\nu_{i n} / \omega_{0}$, would increase. This decrease in the average wave growth is what then decreases the necessary coupling voltage as electrons lose less energy to waves and couple more easily to the thruster plume. We will now determine realistic parameters as inputs to the model.

\section{Experimentally Informed Model Inputs}

Armed with the resulting equation from the previous section, we want to validate the model to determine if it accuractely predicts pressure dependence. To this end, for our case study, we will examine the SPT-100 as this system has one of the most extensive pressure studies to date. ${ }^{29}$ In particular, we will use realistic plasma parameters and physical values from the SPT-100 cathode to see if the model is able to capture the same results as the pressure studies.

During these studies, both cathode-to-ground and plasma potential measurements were taken. From these, the cathode coupling voltage can be inferred. As seen in Figure 3, an exponential decrease is seen in cathode coupling voltage with increasing background pressure. Plotted here is the change in cathode coupling voltage, not the total coupling voltage. 


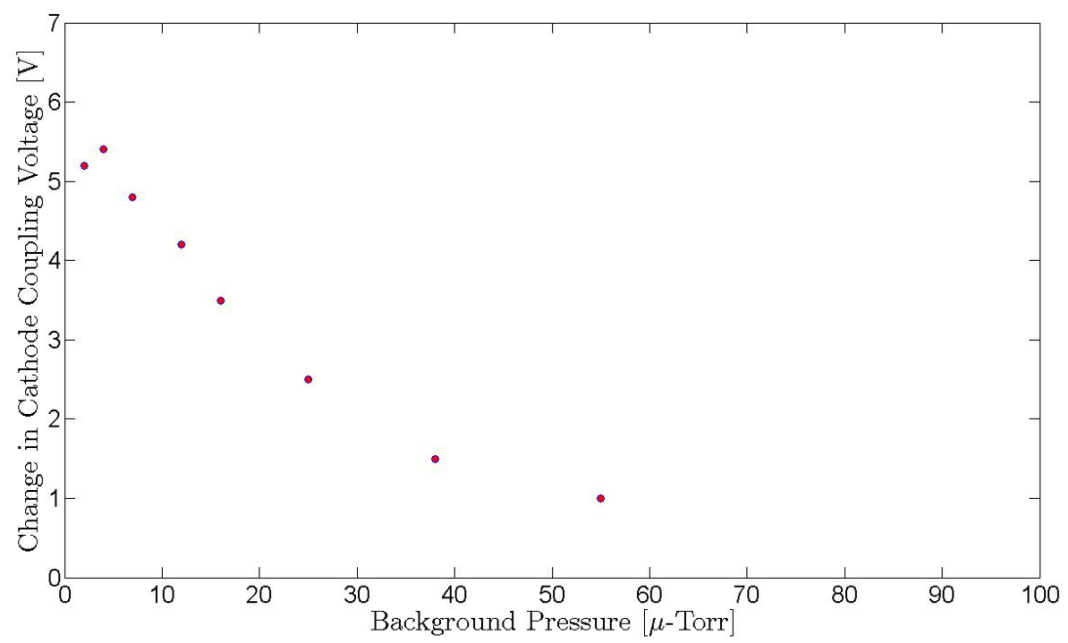

Figure 3: Change in cathode coupling voltage as a function of background pressure. This data was inferred from cathode to ground and plasma potential measurements, not directly measured ${ }^{29}$

While many relevant parameters can be taken from the SPT-100 data, there are some values that were not experimentally measured. Therefore, a curve-fitting algorithm is used to find the values of these parameters for the given model. Then, the found parameters are compared to parameters available in literature for other cathodes to determine whether they are realistic. For the model, we expect $\alpha \times \delta$ to be on the order of $10^{-4}$ to $10^{-6},{ }^{23}$ however since it is not experimentally known, it was left as a free parameter in the fit. The same was done for $\omega_{0}$. The total coupling length is also a parameter that is unknown. It was set to $20 \mathrm{~cm}$ which is a relevant length scale for the thruster. The remaining variables were taken from experimental data. The discharge current through the cathode is,

$$
I_{D}=n_{e} A V_{e} q
$$

where $A$ is the area of the orifice. Equation 15 is used to determine the electron drift velocity. An electron temperature of $2.5 \mathrm{eV}$ and electron density on the order of $10^{13} \mathrm{~cm}^{-3}$ were used based on experimental values. ${ }^{30}$ Based on experimental observation, $T_{i}$ was taken as $0.4 \mathrm{eV} .{ }^{31}$ The ion sound speed was calculated as,

$$
c_{s}=\sqrt{\frac{q T_{e}}{m_{i}}}
$$

where $m_{i}$ is the mass of the xenon ion. The electron thermal velocity, $v_{E}$, was calculated as,

$$
v_{e}=\sqrt{\frac{q T_{e}}{m_{e}}}
$$

The profiles detailed were taken at a discharge current of $4.5 \mathrm{~A}$ and a cathode orifice radius of 0.6 $\mathrm{mm} .{ }^{32}$ The ion speed and the group velocity speed were assumed to be on the order of the ion acoustic speed.

Background pressure is introduced through the constant $\left\langle k_{i}\right\rangle$, as $\nu_{i n}$ is

$$
\nu_{i n}=\sigma_{n} V_{i} n_{n}
$$

where $\sigma_{n}$ is the cross-section with value of $10^{-18} \mathrm{~m}^{2}$ given by Miller ${ }^{33}$ and $n_{n}$ is the neutral density. The background pressure was varied between $1 \times 10^{-7}$ Torr and $1 \times 10^{-3}$ Torr. In order to convert 
the background pressure into a neutral density, the background temperature was assumed to be $300 \mathrm{~K}$ and the ideal gas law was used. The background pressure was added to the neutral density due to the cathode. This was estimated as $10^{19} \mathrm{~m}^{-3}$, which is the same order as the plasma density and the average value of the curve reported by Spektor $^{19}$ for his cathode coupling model.

\section{Results}

Table 1: Model Inputs to Capture Facility Effects

\begin{tabular}{c|c} 
Parameter & Value \\
\hline \hline Electron Temperature & $2.5 \mathrm{eV}$ \\
Ion Temperature & $0.4 \mathrm{eV}$ \\
$\alpha \times \delta$ & $5 \times 10^{-6}$ \\
$\omega_{0}$ & $9.2 \times 10^{5} \mathrm{~Hz}$ \\
Electron density & $5 \times 10^{13} \mathrm{~cm}^{-3}$ \\
Cathode neutral density & $10^{13} \mathrm{~cm}^{-3}$ \\
$x_{f}-x_{0}$ & $20 \mathrm{~cm}$
\end{tabular}

Figure 4 show the results of the model as compared to experimental data inferred from the SPT-100. ${ }^{34}$ The data presented is the change in coupling voltage as a function of pressure, not the absolute value of coupling voltage. The model shows good agreement with the experimental data. The parameter inputs for the model are seen in Table 1. While there is good agreement between the model and the experimental data, we still need to determine whether the fitting parameters, $\omega_{0}$ and $\alpha \times \delta$, are realistic values for the system. Additionally, because experimental values have uncertainty, these fitting parameters will vary within experimental uncertainty. In an attempt to characterize the variation of these parameters with experimental uncertainty, the input electron temperature and electron density were allowed to vary and the parameters necessary for fitting were calculated for a variety of these cases. The electron temperature was allowed to vary between 2.5 and $3 \mathrm{eV}$ while the electron density was allowed to vary between $3 \times 10^{13} \mathrm{~cm}^{-3}$ and $5 \times 10^{13} \mathrm{~cm}^{-3}$. A two-dimensional matrix of potential electron temperature and density values was constructed and the algorithm fit the model to the experimental data for each of the points. The corresponding free parameters can be seen in Table 2. 


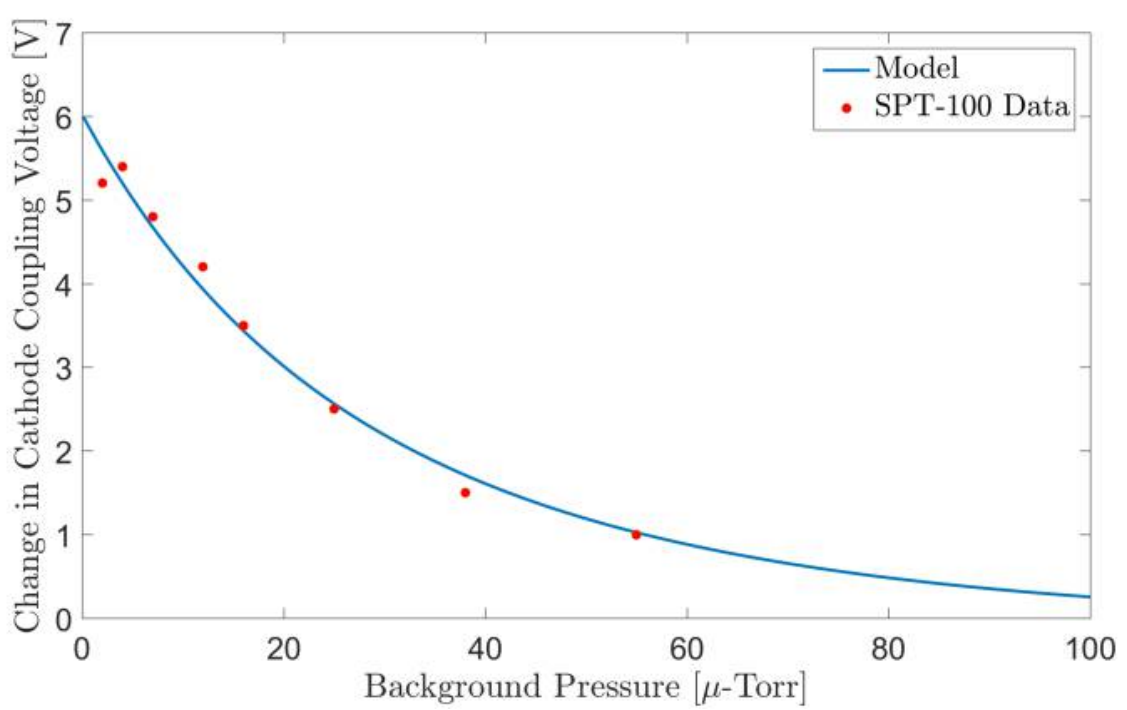

Figure 4: Plot showing the results of simulating equation 13 versus SPT-100 data inferred from Diamant. ${ }^{34}$ The results shown are changes in the coupling voltage not the absolute voltage.

Table 2: Results of Uncertainty Analysis

\begin{tabular}{|c|c|c|c|}
\hline Parameter & Model Value & Model Uncertainty & Value from Literature $^{23}$ \\
\hline \hline$\alpha \times \delta$ & $3.4 \times 10^{-6}$ & $0.1 \times 10^{-6}$ & $10^{-6}-10^{-4}$ \\
\hline$\omega_{0}$ & $3.4 \times 10^{5} \mathrm{~Hz}$ & $2.9 \times 10^{5} \mathrm{~Hz}$ & $5 \times 10^{5} \mathrm{~Hz}$ \\
\hline
\end{tabular}

The results show good agreement between known values for the free parameters suggesting that the model is promising for properly capturing pressure sensitivity on cathode coupling voltage.

\section{Discussion}

In general, the results show that including the ion acoustic turbulence into the anomalous frequency is a potential explanation for the variation of the changing coupling voltage with background pressure. This indicates that with increasing background pressure, the growth of the ion acoustic modes are damped, causing the wave energy to decrease. In turn, this causes the resistivity of the plasma to decrease making it easier for the electrons to couple to the channel, thereby decreasing the cathode coupling voltage. Conversely, it shows that with decreasing background pressure, the electron drift drives a wave that takes energy from the electrons requiring more potential to couple them to the plume of the thruster. This agrees with experimental data suggesting that changes in background facility pressure results in changes in the cathode coupling voltage which in turn likely effects the thruster performance.

Based on Eqn. 14, there are further conclusions that can be drawn. Performance ${ }^{35}$ is known to decrease with decreasing cathode flow fraction, which this model suggests. Neutral density decreases with decreasing cathode flow fraction. Therefore, this model suggests a similar trend as seen with decreasing background pressure for decreasing cathode flow fraction. This is supported by the experimental data. Finally, based on previous models, ${ }^{22}$ it is expected that the wave energy would eventually saturate. This would indicate that at a low enough background density, likely when the cathode plume is dominating the neutral density, the change in coupling voltage would 
be insensitive to further decreases.

To first order, with a smaller coupling voltage, an increased amount of the discharge voltage is used to accelerate the ions in the discharge. This would increase the efficiency of the thruster which is seen by Diamant. ${ }^{34}$ A $6 \mathrm{~V}$ change in coupling voltage, which is seen at $1 \mu$ Torr, and a $300 \mathrm{~V}$ discharge voltage, a typical value for Hall thrusters, would result in a $2 \%$ decrease in voltage utilization efficiency. Thruster performance is known to vary more than $2 \%$, however it does help capture some of the degradation in the total performance with decreasing background pressure. Additionally, there may be further implications on the thruster performance from the cathode coupling voltage changing. A potential limitation of the approach outlined here is an assumption of fixed boundary conditions. However, Nakels et al ${ }^{10}$ saw movement of the acceleration region with background pressure variation and the mechanism behind this is unknown. A change in cathode coupling voltage could result in a change to the boundary conditions of the acceleration region, which would have potential implications on the location of the acceleration region. However, the implications of the cathode-coupling voltage changing are an ongoing research path.

To the correct order of magnitude, which is the accuracy of many experimental values, the model captures the decrease in cathode coupling voltage. However, there are still some open questions. Particularly, the implications of relaxing some of the key assumptions are unknown. Finally, the model needs to be applied to other thrusters to determine its extensibility.

\section{Conclusions}

A one-dimensional quasi-linear model was developed to capture the susceptibility of the cathode coupling voltage to the background facility pressure. Experimental evidence has shown that the magnitude of this parameter increases with decreasing background pressure. The underlying hypothesis is that the onset of ion acoustic turbulence as the background pressure decreases takes energy away from the electrons and makes it harder for them to couple to the Hall thruster channel. Alternatively, the model states that the damping of the ion acoustic modes leads to decreased electron resistivity and coupling voltage magnitude with increasing background pressure. The model agrees well with experimental data from the SPT-100. The results show up to a $2 \%$ decrease in voltage utilization by decreasing the pressure. Even though the cathode coupling voltage only represents a small portion of the total performance, this is a promising first-principles model of the effect. Open-questions remain including the extensibility to other thrusters and the implications of the cathode-coupling voltage changes on the boundary condition for the acceleration region.

\section{Acknowledgments}

The authors would like to acknowledge funding provided by the NASA Space Technology Research Fellowship grant number NNX15AQ43H and Air Force Office of Scientific Research grant number F043602-01.

\section{References}

\footnotetext{
${ }^{1}$ Hofer, R. R., Development and characterization of high-efficiency, high-specific impulse xenon Hall thrusters, University of Michigan, 2004.

$>^{2}$ Mikellides, I. G., Katz, I., Hofer, R. R., and Goebel, D. M., "Magnetic shielding of a laboratory Hall thruster. I. Theory and validation," Journal of Applied Physics, Vol. 115, No. 4, 2014, pp. 043303.
} 
${ }^{3}$ Koppel, C. R. and Estublier, D., "The SMART-1 Hall effect thruster around the moon: In flight experience," 29th International electric propulsion conference, 2005, p. 119.

${ }^{4}$ Oh, D. Y., Goebel, D., Polanskey, C., Snyder, S., Carr, G., Collins, S. M., Lantoine, G., Landau, D., ElkinsTanton, L., Lord, P., et al., "Psyche: Journey to a Metal World," Small, Vol. 30, 2016, pp. 50.

${ }^{5}$ Hofer, R. R., Randolph, T. M., Oh, D. Y., Snyder, J. S., and de Grys, K. H., "Evaluation of a 4.5 kw commercial hall thruster system for nasa science missions," AIAA paper, Vol. 4469, 2006, pp. 9-12.

${ }^{6}$ Brophy, J. R., Barnett, J., Sankovic, J., and Barnhart, D., "Performance of the stationary plasma thruster: SPT-100," 28th AIAA Joint Propulsion Conference, Nashville, TN, 1992.

${ }^{7}$ Randolph, T., Kim, V., Kaufman, H., Kozubsky, K., Zhurin, V., and Day, M., "Facility effects on stationary plasma thruster testing," 23rd International Electric Propulsion Conference, 1993, pp. 13-16.

${ }^{8}$ Hofer, R. R. and Anderson, J. R., "Finite Pressure Effects in Magnetically Shielded Hall Thrusters," 50th AIAA/ASME/SAE/ASEE Joint Propulsion Conference, 2014, p. 3709.

${ }^{9}$ Huang, W., Kamhawi, H., and Haag, T., "Facility Effect Characterization Test of NASAs HERMeS Hall Thruster," 52nd AIAA/SAE/ASEE Joint Propulsion Conference, 2016, p. 4828.

${ }^{10}$ Nakles, M. and Hargus, W., "Background pressure effects on internal and near-field ion velocity distribution of the bht-600 hall thruster," 44th AIAA/ASME/SAE/ASEE Joint Propulsion Conference E Exhibit, 2008 , p. 5101.

${ }^{11}$ Spektor, R. and Tighe, W. G., "Laser Induced Fluorescence Measurements in a Hall Thruster as a Function of Background Pressure," 52nd AIAA/SAE/ASEE Joint Propulsion Conference, 2016, p. 4624.

${ }^{12}$ Hofer, R. R., Peterson, P. Y., and Gallimore, A. D., "Characterizing vacuum facility backpressure effects on the performance of a Hall thruster," IEPC-01-045, 27th International Electric Propulsion Conference, Pasadena, CA, 2001.

${ }^{13}$ Huang, W., Kamhawi, H., and Haag, T., "Effect of background pressure on the performance and plume of the hivhac hall thruster," 2013.

${ }^{14}$ Byers, D. and Dankanich, J. W., "A review of facility effects on Hall effect thrusters," 31st International Electric Propulsion Conference, 2009.

${ }^{15}$ Frieman, J. D., King, S. T., Walker, M. L., Khayms, V., and King, D., "Role of a Conducting Vacuum Chamber in the Hall Effect Thruster Electrical Circuit," Journal of Propulsion and Power, Vol. 30, No. 6, 2014, pp. 1471-1479.

${ }^{16}$ Frieman, J. D., Walker, J. A., Walker, M. L., Khayms, V., and King, D. Q., "Electrical Facility Effects on Hall Thruster Cathode Coupling: Performance and Plume Properties," Journal of Propulsion and Power, Vol. 32, No. 1, 2015, pp. 251-264.

${ }^{17}$ Crofton, M. W. and Pollard, J., "Thrust augmentation by charge exchange," 49th AIAA/ASME/SAE/ASEE Joint Propulsion Conference, 2013, p. 4131.

${ }^{18}$ Kamhawi, H., Huang, W., Haag, T., and Spektor, R., "Investigation of the Effects of Facility Background Pressure on the Performance and Operation of the High Voltage Hall Accelerator," 50th AIAA/ASME/SAE/ASEE Joint Propulsion Conference, 2014, p. 3707.

${ }^{19}$ Spektor, R., T. W. S. P. and K, B., "Facility Effects on Hall Thruster Performance Through Cathode Coupling," 34th International Electric Propulsion Conference, 2015.

${ }^{20}$ Mikellides, I., Katz, I., Goebel, D., and Polk, J., "Theoretical Modeling of a Hollow Cathode Plasma for the Assessment of Insert and Keeper Lifetimes," 41st AIAA/ASME/SAE/ASEE Joint Propulsion Conference, 2005.

${ }^{21}$ Mikellides, I., Katz, I., Goebel, D., and Polk, J., "Theoretical model of a hollow cathode insert plasma," 40th AIAA/ASME/SAE/ASEE Joint Propulsion Conference and Exhibit, 2004, p. 3817.

${ }^{22}$ Jorns, B., Lopez Ortega, A., and Mikellides, I. G., "First-principles Modelling of the IAT-driven Anomalous Resistivity in Hollow Cathode Discharges I: Theory," 52nd AIAA/SAE/ASEE Joint Propulsion Conference, 2016, p. 4626.

${ }^{23}$ Lopez Ortega, A., Mikellides, I. G., and Jorns, B., "First-principles modeling of the IAT-driven anomalous resistivity in hollow cathode discharges II: Numerical simulations and comparison with measurements," 52nd AIAA/SAE/ASEE Joint Propulsion Conference, 2016, p. 4627.

${ }^{24}$ Dodson, C., Perez-Grande, D., Jorns, B., Goebel, D. M., and Wirz, R. E., "Laser-induced Fluorescence Measurements of Energetic Ions in a 100-A LaB6 Hollow Cathode Plume," 52nd AIAA/SAE/ASEE Joint Propulsion Conference, 2016, p. 4838.

${ }^{25}$ Mikellides, I. G., Katz, I., Goebel, D. M., and Polk, J. E., "Hollow cathode theory and experiment. II. A twodimensional theoretical model of the emitter region," Journal of Applied Physics, Vol. 98, No. 11, 2005, pp. 113303.

${ }^{26}$ Lopez Ortega, A. and Mikellides, I. G., "The importance of the cathode plume and its interactions with the ion beam in numerical simulations of Hall thrusters," Physics of Plasmas, Vol. 23, No. 4, 2016, pp. 043515.

${ }^{27}$ Sagdeev, R. Z. and Galeev, A. A., "Nonlinear plasma theory," Nonlinear Plasma Theory, New York: Benjamin, $1969,1969$.

13 of 14 
${ }^{28}$ Jorns, B. A., Dodson, C., Goebel, D. M., and Wirz, R., "Propagation of Ion Acoustic Wave Energy in the Plume of a High-Current LaB6 Hollow Cathode," Physical Review E, 2017 (Accepted).

${ }^{29}$ Diamant, K., Spektor, R., Beiting, E., Young, J., and Curtiss, T., "The effects of background pressure on Hall thruster operation," 48th AIAA/ASME/SAE/ASEE Joint Propulsion Conference E Exhibit, 2012, p. 3735.

${ }^{30}$ Liu, H., Li, M., Ning, Z., Ren, J., Tang, H., Yu, D., Demidov, E. V., Eliseev, S. I., and Kudryavtsev, A. A., "2-D modeling of orificed hollow cathodes of stationary plasma thrusters spt-100," IEEE Transactions on Plasma Science, Vol. 43, No. 12, 2015, pp. 4024-4033.

${ }^{31}$ Goebel, D. M. and Katz, I., Fundamentals of electric propulsion: ion and Hall thrusters, Vol. 1, John Wiley \& Sons, 2008.

${ }^{32}$ Garner, C., Brophy, J., Polk, J., and Pless, L., "A 5,730-hr cyclic endurance test of the SPT-100," 31st Joint Propulsion Conference and Exhibit, 1995, p. 2667.

$\checkmark{ }^{33}$ Miller, J. S., Pullins, S. H., Levandier, D. J., Chiu, Y.-h., and Dressler, R. A., "Xenon charge exchange cross sections for electrostatic thruster models," Journal of Applied Physics, Vol. 91, No. 3, 2002, pp. 984-991.

${ }^{34}$ Diamant, K. D., Liang, R., and Corey, R. L., "The effect of background pressure on SPT-100 Hall thruster performance," 50th AIAA/ASME/SAE/ASEE Joint Propulsion Conference, 2014, p. 3710.

${ }^{35}$ Jameson, K. K., Investigation of hollow cathode effects on total thruster efficiency in a $6 \mathrm{~kW}$ Hall thruster, University of California, Los Angeles, 2008. 